\title{
LA COMUNICACIÓN INSTITUCIONAL-INTERCULTURAL EN EL ESPACIO PÚBLICO MADRILEÑO: UNA NUEVA CATEGORÍA SOCIO- ANTROPOLÓGICA EN CONSTRUCCIÓN
}

\author{
INSTITUTIONAL-INTERCULTURAL CORPORATE COMMUNICATION IN \\ MADRID: A NEW SOCIOANTHROPOLOGICAL CATEGORY
}

\author{
Carmen Gaona \\ Esther Martínez \\ Ricardo Vizcaíno \\ Universidad Rey Juan Carlos, Madrid. España/Spain \\ carmen.gaona@urjc.es \\ esther.martinez.pastor@ujc.es \\ ricardo.vizcaino@urjc.es
}

Recibido/Received: 25/02/2011

Aceptado/Accepted: 01/09/2011

\section{RESUMEN}

El artículo propone una categoría comunicacional que bajo la denominación de "comunicación institucional-intercultural" incluiría aquellos fenómenos comunicativos en donde, con la participación de las Administraciones Públicas, el desarrollo de espacios concretos y de una imagen de marca se pretende el desarrollo de políticas de integración cultural con inmigrantes. Esta nueva categoría surge tras una investigación desarrollada en los Centros de Participación e Integración del Inmigrante (CEPI), un concepto integrador creado en Madrid y considerado único en Europa.

\section{PALABRAS CLAVE}

Inmigración, integración, comunicación institucional-intercultural, CEPI.

\section{SUMARIO}

Introducción. 1. Precisiones conceptuales: de la comunicación intercultural a la comunicación institucional-intercultural. 2. Servicios, distribución geográfica y gestión. 3. Presentación conceptual de los CEPI. 4. Conclusiones. Bibliografia.

\section{ASBTRACT}

The text suggests a new communicational category named "corporate-intercultural communication" in order to the integration of immigrants into the society, which include: Public Administration, specific places and well-know brand. This new category is the result of a research in the Centre for Participation and Integration of Immigrant (CEPI), a unique reputed policy of integration all over Europe which is developed in Madrid.

\section{KEYWORDS}

Immigration, integration corporate-intercultural communication, CEPI. 


\section{CONTENTS}

Introduction. 1. Concepts: intercultural communications and corporate-intercultural communication. 2. Services, geographical distribution and management. 3. Presentation Conceptual introduction to the CEPI. 4. Conclusions. References.

\section{INTRODUCCIÓN}

Hemos vertebrado nuestra investigación, en la corroboración de una nueva categoría comunicativa: la comunicación institucional-intercultural, ideada por el equipo investigador y autores de este artículo. En toda la bibliografía existente, tanto en la Sociología de la comunicación como en las Teorías de la Comunicación, el objeto de estudio central ha sido la comunicación intercultural. Nuestra nueva categoría parte de esta última, con la que comparte características comunes, pero se resignifica en unos escenarios urbanos concretos: los CEPI (Centro de Participación e Integración de Inmigranes de la Comunidad de Madrid). De ahí que iniciemos este capítulo con una puntualización epistemológica sobre el concepto de "comunicación intercultural-institucional", seguido de una presentación y descripción de dichos centros.

\section{PRECISIONES CONCEPTUALES: DE LA COMUNICACIÓN INTER- CULTURAL A LA COMUNICACIÓN INSTITUCIONAL-INTERCULTURAL}

El objeto de este capítulo es identificar, definir y delimitar las particularidades de la comunicación institucional-intercultural. Para ello, entendemos necesario comenzar con una breve contextualización de la comunicación intercultural, como concepto del que deriva nuestro eje conceptual.

Son incontables las múltiples propuestas que definen la "comunicación intercultural", si bien como punto de encuentro de todas de ellas, podemos decir, que la comunicación intercultural recoge los intercambios comunicativos intersubjetivos e interpersonales que se establecen entre pueblos con diferentes sistemas socioculturales y/o la comunicación entre miembros de diferentes subsistemas (por ejemplo grupos étnicos, grupos nacionales o grupos religiosos) dentro del mismo sistema sociocultural. Ésta es una definición que incide en las características comunicativas, para evitar la dialéctica presente en los discursos identitarios nacionales y culturales, con los que suele explicar la comunicación intercultural.

En los años 60, cuando surgen en un contexto norteamericano la producción académica sobre la comunicación intercultural, este interés no sólo responde a una nueva temática marcada desde los escenarios académicos, sino que nace de un doble empuje: uno endógeno y el otro inicialmente exógeno. La motivación endógena, viene explicada por la reivindicación de algunas minorías de su propia cultura, como es el caso de los afronorteamericanos. En contrapartida, otros fenómenos sociales potenciaron también la necesidad de iniciar este tipo de estudios, fenómenos tales como las guerras en el sudeste asiático que supuso no sólo un contacto con las culturas de estos países, sino también que una gran cantidad de personas de Camboya, Laos y Vietnam se refugiaran en los Estados Unidos de América. A estos flujos migratorios hay que añadir los de los países latinoamericanos" (Sendín y Gaona, 2009:93-94). Entra de este modo a la escena académica el interés por la comunicación intercultural, aunque evidentemente ésta ha existido desde el inicio de la 
historia de la humanidad, puesto que los procesos migratorios (como una de las principales dinámicas sociodemográficas que posibilitan el contacto cultural) han existido desde nuestros orígenes, sin obviar ni mucho menos los contactos culturales ocasionados por las grandes rutas comerciales, tan presentes todavía en nuestro imaginario colectivo.

El objetivo de nuestro artículo es reflexionar sobre la comunicación institucional-intercultural, una vez presentado de forma breve la comunicación intercultural, como la antecesora y gran puntal de nuestra categoría, pasamos a exponer nuestro itinerario investigador que nos condujo y concluyó en esta nueva forma comunicativa. En el caso concreto que nos ocupa, durante los años 2007 y 2008, el Observatorio de las Realidades Sociales y la Comunicación, del que son miembros los autores, bajo la financiación de la Consejería de Inmigración de la Comunidad Autónoma de Madrid (CAM), puso en marcha una investigación enmarcada en el contexto de unos nuevos dispositivos de esta Consejería: los "Centros de Participación e Integración del Inmigrante" (CEPI). Los CEPI quedaban contemplados en el "Plan de Integración 2006-2008" de la CAM, cuyos ejes esenciales fueron: el encuentro de españoles-inmigrantes, el conocimiento recíproco, la integración y la pacífica convivencia. El propósito de los CEPI radica en "propiciar el encuentro y el conocimiento recíproco entre la población inmigrada y la autóctona" (...) "poniendo en funcionamiento los Centro de Participación e Integración de los Inmigrantes" (Vizcaíno-Laorga, Martínez y Gaona, 2009:111). Tal y como se afirma en dicho Plan "estos Centros serán espacios de convivencia desde el que se propiciará el acercamiento mutuo. Se promocionarán desde los mismos cuantas actuaciones se consideren de interés para producir al integración y la convivencia pacífica desde la pluralidad" (Plan Integración 20062008: CAM, 127).

Dichos centros pretendían dar un paso más en las políticas de atención al fenómeno migratorio impulsadas en la Comunidad de Madrid, ampliando las prestaciones ofrecidas en los antecesores de los CEPI, los "Centros de Atención Social al Inmigrante" (CASI), básicamente centrados en una atención asistencial. Con los CEPI se pretendía crear unos espacios culturales de encuentro y convivencia entre población de origen madrileño y población de origen inmigrante, en los que activar nuevas dinámicas de integración.

Un requisito básico, o mejor dicho, el punto de arranque de toda integración, son las prácticas comunicativas, que puesto que son protagonizadas por ciudadanos procedentes de diversos orígenes culturales se categorizaron, en un primer nivel, como dinámicas de comunicación intercultural. No obstante, tras el trabajo empírico y posterior análisis, descubrimos que estábamos ante un nuevo tipo de comunicación intercultural, que categorizamos con el nombre de "comunicación institucional-intercultural". El hecho de crear esta nueva categoría vino suscitado y cimentado, ante el hallazgo en las prácticas comunicativas analizadas de ciertas particularidades, así como nuevos elementos que personalizaban un tipo concreto de comunicación en los nuevos escenarios urbanos que constituyen los CEPI.

\subsection{Las particularidades de la comunicación institucional-intercultural}

Las características de la comunicación institucional-intercultural son las siguientes: aparición de un nuevo agente activo en la comunicación intercultural que son las administraciones públicas a través de las políticas de integración; ubicación en unos nuevos espacios urbanos mediante los CEPI, "escenarios en tránsito"; vinculación con los valores de imagen de marca; relación con la dinámica adaptativa; asociación con el "interés general"; y unión con la diversidad cultural. 
a. 'Un nuevo agente activo en la comunicación intercultural: las administraciones públicas a través de las políticas de integración'

Las definiciones sobre comunicación intercultural focalizan su interés en la comunicación interpersonal y las investigaciones centradas en este tema se centran en establecer y comprender qué sucede cuando los seres humanos de diferentes culturas se reúnen, interactúan e intentan resolver los problemas en distintas interrelaciones, dejando de lado otros sujetos que intervienen en la comunicación. En nuestra nueva categoría se introduce un nuevo actor que está presente en la comunicación intercultural, ocupando un espacio y función concreta: las Administraciones Públicas. Este nuevo actor institucional alterna una multi-proximidad: de un lado, debe de estar en constante diálogo con los diferentes grupos culturales constitutivos de la sociedad y, de otro lado, elabora y construye mensajes a través de "las políticas de integración". De este modo, podemos considerar que la Administración Pública es un nuevo agente activo en la co-implicación urbana por la construcción de una sociedad diversa y plural desde el punto de vista cultural y comunicacional.

Un objetivo político-social dentro de las agendas de las Administraciones Públicas es el de diseñar políticas de integración para lograr una sociedad plural y la implementación de nuevos dispositivos como herramientas para lograr una diversidad social. La consecución de estos fines supone políticas plurales dentro de una misma área, aplicables al campo educativo, de la salud, así como de las políticas de integración en temas de inmigración. En el campo que nos ocupa, identificamos diferentes estrategias políticas de integración diseñadas por la Consejería de Inmigración de la Comunidad de Madrid concretadas en diferentes acciones, con distintos recursos y aplicadas a existentes o nuevos dispositivos. En este caso, nos hemos centrado en uno de ellos: los CEPI.

\section{b. 'La comunicación institucional-intercultural en unos nuevos espacios urbanos: los CEPI, "escenarios en tránsito"”}

La comunicación intercultural no precisa de un escenario específico, ni se da en una misma ágora. La comunicación se produce en el contacto entre dos personas de diferentes grupos culturales en un lugar cualquiera del espacio urbano: en un supermercado, en la puerta de un colegio, etc. Mientras que la comunicación institucional-intercultural requiere de la creación de nuevos escenarios urbanos, planificados para que se conviertan en escenarios cotidianos de interculturalidad y en parte integrante de la ciudad. Esta necesidad de nuevos escenarios inherente a la comunicación institucional/ intercultural, viene argumentada por el contacto de grupos culturales relativos a nuevos ciudadanos-inmigrantes que tanto si están recién llegados al territorio español, como si ya llevan varios meses o años en España, todavía están en ese proceso de construcción de una nueva identidad en la que confluyan tanto elementos culturales de su grupo de origen, como elementos culturales de la sociedad de acogida. De alguna manera, los inmigrantes están con una identidad en tránsito tal y como acuñó Clifford (1992:109) "todas las personas están en cierta medida permanentemente en tránsito... No tanto ¿de dónde vienes?, sino ¿entre dónde estás?”. En el caso, de los CEPI éstos son "escenario en tránsito", inclusive esto se trasluce en su denominación. En cierto modo, los CEPI son nombrados delatando esa situación de paso: CEPI Hispano-Marroquí, CEPI Hispano-Ecuatoriano, etc. Nombres que se relacionan con ese segundo interrogante, ¿entre dónde estás? Estos nuevos espacios, los CEPI, son una clara implementación de políticas concretas de integración derivadas de las "políticas de integración" impulsadas desde la Consejería de Inmigración de la Comunidad de Madrid, como agente social. 


\section{c. 'La comunicación institucional-/intercultural: valores e Imagen de marca'}

La Administración Pública refuerza símbolos en su comunicación institucional-intercultural para que a un mismo tiempo sean identificados, reconocidos y diferenciados por ellos mismos y por los demás. En el diálogo de los poderes públicos deben estar presentes e identificados: el Gobierno, los ciudadanos autóctonos y los inmigrantes. Éstos se identifican y son reconocibles por sus símbolos propios, así como deben recrearse conjuntamente en los "espacios comunes" en los que armónicamente conviven. De este modo es comprensible que la Administración Pública como "nuevo agente activo en la comunicación intercultural" potencie de forma indirecta su presencia como sujeto mediador y construya un posicionamiento más que positivo de su participación ensalzando su imagen de marca de "gobierno-institución". Su objetivo es reforzar su "marca", la Comunidad de Madrid, y generar un clima de confianza con cada uno de ellos. Esto se consigue a través de una "comunicación funcional", porque se informa sobre las funciones y servicios de las Administración, en este caso de la existencia de los CEPI, y de la "comunicación simbólica", reforzando la imagen corporativa de la Comunidad Autónoma de Madrid.

La imagen de marca es "la estructura mental de la organización que se forman los públicos, como resultado del procesamiento de toda la información relativa a la organización" (Capriotti, 1999:29), es decir, el conjunto de significados que una persona asocia a una organización. Se trata de ideas utilizadas para describir o recordar a una organización. Dowling (1994:8) la define como la impresión total (creencias y sentimientos) que una organización genera en la mente de los públicos, en concreto en sus "imaginarios sociales". El imaginario social "está configurado por los sentidos con los que las personas corrientes, nosotros mismos, "imaginamos" nuestro entorno social. (...) pero la grandeza del imaginario social radica en que éste es la concepción colectiva que posibilita las prácticas comunes y un sentimiento ampliamente compartido de legitimidad" (Gaona, 2009:68-70). Nuestra propuesta es que poco a poco, por acción de la "comunicación institucional-intercultural", la imagen de marca de la Administración Pública mediante una paulatina interiorización del sujeto, se adhiere al imaginario colectivo de los usuarios de los CEPI.

Los mensajes difundidos para este fin deben adaptarse a las necesidades y cambios sociales como parte de construcción social de la realidad. Este proceso debe consolidarse como acuñó Villafañe por la buena reputación de una institución hacia sus públicos.

Capriotti (1999:97) señala que pueden distinguirse tres fuentes de información que intervienen en la construcción de la imagen: los medios de comunicación masivos, las relaciones interpersonales (influencia de los grupos de referencia y los líderes de opinión) y la experiencia personal (fuente de información más decisiva aunque su influencia puede estar mediatizada por las dos fuentes anteriores). En el caso de los CEPI la imagen de marca se construye a través de las relaciones interpersonales y de la experiencia personal. Esta comunicación puede ser duramente criticada por suscitar el "autobombo" con fines electorales del gobierno de turno que aproveche la coyuntura social. Sin embargo, la creación de los CEPI como lugares de encuentro, debería ser claramente identificada como acción de la Comunidad de Madrid, dado que son los promotores de los mismos, su financiación es pública y son unas de las acciones precisas para el logro del bienestar social. Y aunque, en ocasiones el mensaje simbólico prevalezca sobre el de referencia (Caro, 2007) debemos tener presente que uno de los intereses de la comunicación institucional es el interés general y en este caso concreto está más que justificado. Cuestión aparte sería las críticas a los procesos administrativos de gestión de los mismos que no es objeto de nuestro estudio. 


\section{d. 'La comunicación institucional-intercultural en simbiosis con el "interés general"'}

El interés general es otra de las características de la comunicación institucional-intercultural. De acuerdo con Parada toda actividad administrativa debe responder al interés común "que, aunque no beneficie a la totalidad de la comunidad, sí favorece al menos a una fracción importante de sus miembros" (Parada, 1998:438). Por ello, y como apunta Martínez Pastor, el beneficio de la gran mayoría se cumple en interés general (2010:150). Por ello, esta características es propia de la naturaleza de esta comunicación de índole pública porque, de un lado el comunicante es la Administración Pública y de otro lado el mensaje es de interés general porque persigue la convivencia entre diferentes culturas y el respeto mutuo. En este sentido, "los CEPI son espacios para cualquier ciudadano que quiera acudir a ellos para realizar una actividad o solicitar algún tipo de ayuda, pues buscan que los intereses de todos puedan ser cubiertos en estos dispositivos lógicamente, dentro de sus posibilidades y competencias" (Vizcaíno-Laorga, Martínez y Gaona, 2008:19).

\section{e. 'La comunicación institucional-.intercultural como puente con la diversidad cultural'}

Esta comunicación promovida por la Administración Pública pretende encontrar herramientas constructoras de una sociedad plural, que precisamente es uno de los objetivos principales de la comunicación intercultural. Este trabajo se manifiesta a través de las políticas de integración en las que se plantean la heterogeneidad de lo público y, a un mismo tiempo, la visibilidad de la diversidad social. Un claro ejemplo son los CEPI en los que la comunicación institucional/intercultural parte de la Administración en cuanto comunicante y activador de los CEPI como escenarios de tránsito artificiales que muestran la sociedad plural y las diferentes maneras de actuación de los poderes públicos para gestionar las necesidades de cada momento. Aunque, inevitablemente y, quizá de forma también estratégicamente planificada promocionan la imagen de marca de la Comunidad de Madrid.

Partiendo de esta delimitación teórica del concepto de la comunicación institucionalintercultural pasamos a realizar una descripción de los servicios ofertados en los CEPI, su ubicación geográfica y la gestión de los mismos.

\section{SERVICIOS, DISTRIBUCIÓN GEOGRÁFICA Y GESTIÓN}

Los servicios que ofrecen los CEPI se centran en estas funciones básicas: ayuda psicológica, jurídica, mediación social, cursos de formación para el empleo, de ocio, artísticos y apoyo a menores, así como otros cursos de idiomas e informática (Vizcaíno- Laorga, Martínez y Gaona, 2008:52) dirigidos tanto a autóctonos como a inmigrantes, pese a que en la investigación tanto los datos cuantitativos como los cualitativos mostraban cómo la denominación de los CEPI se percibía vinculada estrechamente con los usuarios de los centros, de manera que en el CEPI-boliviano los usuarios eran mayoritariamente de esta nacionalidad con escasa o nula participación de nacionales y otras nacionalidades (VizcaínoLaorga, Martínez y Gaona, 2008:62).

La distribución de los CEPI se produce por toda la Comunidad de Madrid. La mayoría de ellos están ubicados en zonas en las que hay un predominio de inmigrantes, y la denominación del CEPI responde al predominio de una nacionalidad. Actualmente hay 17 CEPI de distinta nacionalidades, y la Comunidad de Madrid delega su gestión a través de un concurso público mediante un Pliego de cláusulas administrativas particulares en el que 
diferentes asociaciones y organizaciones no gubernamentales son encargadas de gestionar los CEPI de la Comunidad Autónoma de Madrid (Vizcaíno-Laorga, Martínez y Gaona, 2009:113).

Cuadro 1: Relación y localización de los CEPI

\begin{tabular}{|l|l|}
\hline CEPI & Ubicación \\
\hline Cepi Hispano- Americano & $\begin{array}{l}\text { C/ Miguel Fleta, 11 C.P. 28037 Madrid. Metro Hermanos García } \\
\text { Noblejas, salida Hermanos García Nobleza }\end{array}$ \\
\hline Cepi Hispano- Ecuatoriano & C/ Jaén, 15 y C/ San Antonio, 4, 28020, Metro: Alvarado \\
\hline Cepi Hispano- Peruano & $\begin{array}{l}\text { Bulevar de Cervantes-Plaza Pizarro, 3, bajo } \\
\text { Majadahonda }\end{array}$ \\
\hline Cepi Hispano- Rumano & C/ Goya n ${ }^{\circ}$ 5 LOCAL $\square 28807$ Alcalá de Henares, (Madrid) \\
\hline Cepi Hispano- Colombiano & C/ Ronda de Segovia, 34, Metro Puerta de Toledo \\
\hline Cepi Hispano- Africano & Manuel Marañón, 13, Metro Arturo Soria \\
\hline Cepi Hispano- Rumano & $\begin{array}{l}\text { Corredor de Henares } \\
\text { Travesía del Curtidor, 1. }\end{array}$ \\
\hline Cepi Hispano- Dominicano & C/Nuestra Señora del Carmen, 17. Madrid \\
\hline Cepi Hispano- Marroquí & C/Colegiata n 10 y C/ Duque de Rivas 8 Metro Lavapiés \\
\hline Cepi Hispano- Búlgaro & C/ Enrique Velasco11 y C/ Picos de Europa 5 \\
\hline Cepi Hispano-Americano & Avda. de España, 8, 28700 San Sebastián de los Reyes (Madrid) \\
\hline Cepi Hispano- Ecuatoriano Arganzuela & Calle Arquitectura n ${ }^{\circ}$ 20 metro Delicias/Acacias \\
\hline Cepi Hispano- Paraguayo & $\begin{array}{l}\text { Calle Luís Cabrera 29 (esquina General Zabala) Metro Prosperidad, } \\
\text { Línea 4 }\end{array}$ \\
\hline Cepi Hispano- Centroamericano & $\begin{array}{l}\text { Calle Fray Ceferino González, 4, Metro: Latina (línea 5), Lavapiés, } \\
\text { Embajadores o Tirso de Molina (línea 3). }\end{array}$ \\
\hline Cepi Hispano- Ucraniano & C/ Antonia Lancha, 50 $\square$ Metro: Plaza Elíptica (L6 y 11) y Opañel (L6) \\
\hline Cepi Hispano- Colombiano Villaverde & $\begin{array}{l}\text { C/ Travesía de tordegrillos, Local Semiesquina Paseo de los } \\
\text { Ferroviarios, 67-69 }\end{array}$ \\
\hline Cepi Hispano- Americano Sur & C/ Esteban Terradas, 15 Leganés, Metro Severo Ochoa \\
\hline & \\
\hline
\end{tabular}

Fuente: elaboración propia

\section{PRESENTACIÓN CONCEPTUAL DE LOS CEPI}

\subsection{CEPI: ¿Espacios liminales/liminoides de comunicación intercultural?}

En la producción científica existe una tradición tanto teórica como empírica de análisis sobre los "espacios liminales y liminoides de comunicación intercultural" en los que podemos observar múltiples prácticas cotidianas de diseños cotidianos de interculturalidad desde diversos niveles de diálogo hasta alcanzar otras escalas de también diversas gradaciones de conflicto social. Atendiendo a tal diversidad gradual de escalas de "cosmopolitismo" hablamos de viejos procesos de aculturación (imitación de los modelos culturales dominantes), o nuevos procesos de adaptación e interculturalización como serían por ejemplo de procesos de transculturalidad hibridizada, entre la diversidad de dinámicas interculturales. Los CEPI son interpretados en nuestra investigación no como "espacios liminales y liminoides" sino como "escenarios en tránsito", y como tales tienen tres potencialidades implícitas, basándonos en las tres zonas de calidad señaladas por Rico Lie.

Primero (coexistencia aislada): como espacios en un estado de coexistencia cultural Lie (2002) define este primer estado de liminalidad, desde un punto de vista de contacto comunicativo como "la co-presencia de islas culturales bastante aisladas" en tanto que 
estamos en los primeros contactos de las personas desterritorializadas caracterizados Nuestra experiencia empírica en los CEPI constata esta mínima fluidez comunicativa inicial, ante las desconfianzas e inseguridades. Los CEPI como espacio de contacto cultural: en este caso, los CEPI simplemente demuestran elementos de diferentes culturas. Estos elementos se presentan uno junto a otro. El estado se marca con la co-presencia de islas culturales bastante aisladas. Se puede mostrar una forma pasiva de comunicación entre los elementos globales, locales, pero no se produce una interacción orientada al cambio significativa, ni una negociación. Se busca comunicar actores/elementos, sean éstos ciudadanos plurales, mobiliario urbano, publicidad, alimentos, costumbres y tradiciones, etc., de forma estática de hibridez; es decir, puede que se produzca una reterritorialización individual de los elementos por separado, pero no se produce demasiada negociación entre los elementos.

Segundo (negociación): como espacios en un estado de negociación intercultural. En este estado sí que se establecen niveles de interacción óptimos para iniciar las negociaciones comunicativas encaminadas hacia "formas dinámicas y participativas de hibridez" (Rico Lie, 2002:18). Sobre este hecho hemos de puntualizar cómo desde nuestra concepción de ciudad vivida, las identidades se labran en los "escenarios", y en estos escenarios interaccionan influencias tanto locales como globales, pues en nuestro análisis nunca podemos perder de vista el papel de los mass media y de las TIC, en las sociedades actuales.

Los CEPI son espacios creados para el consumo cultural y el entretenimiento, y la primera negociación constatada es hecha con la intencionalidad de alcanzar estas gratificaciones de consumo. De este modo, técnicos y usuarios de los CEPI, tuvieron que "negociar" (evidentemente utilizamos este concepto desde un punto de vista de práctica comunicativa), centrando sus mensajes intercambiados, sobre preferencias e intereses de talleres y actividades. Se produce una búsqueda de actividades que satisfagan a los usuarios, cuando éstos abiertamente no lo comunican a los técnicos de los CEPI, de una forma directa y natura, tal y como se constató en las entrevistas realizadas en el trabajo de campo.

Los CEPI como estado de negociación intercultural. En este caso, sí que existe una forma activa de interacción entre los distintos actores/elementos. Interacción centrada en la negociación, hacia una forma dinámica y participativa de diversidad cultural. Es cuando las acciones se concentran hacia el diálogo, la aceptación y el respeto mutuo, pero también es cuando el diálogo deja de ser individual. El diálogo/negociación se establece entre sectores grupales, adquiriendo mayor protagonismo las administraciones públicas.

Tercero (creación): como espacios en un estado de transformación intercultural. La igualdad junto con altos niveles de negociación participativa de formación de formas culturales, serían los dos elementos principales de Rico Lie para este tercer estadio. Sin entrar en el discurso de la idea de igualdad que se equipara al estado de comunitas de Turner, lo más significativo desde nuestro punto de vista sería el hecho de que los diferentes elementos culturales empiezan a ser conocidos, aceptados, compartidos y vividos, un requisito básico para empezar a construir una comunicación intercultural eficaz, previa a establecer acciones interculturales. El CEPI como espacio de transformación intercultural. Este sería el estado ideal no sólo de los CEPI, sino de cualquier espacio intercultural, en el que se perdería ese estatus de "escenario en tránsito", al haberse constituido una identidad cultural compartida por los diferentes grupos culturales de una forma negociada participativa de formas $\mathrm{y}$ elementos culturales de origen local y global. Presentamos este tercer estado a nivel teórico, puesto que en modo alguno se constató en la investigación, un CEPI que hubiese alcanzado este estado. 


\subsection{CEPI: ¿actores globales o actores locales?}

La segunda delimitación epistemológica de los CEPI, se centra en su identidad local o global. Para responder a esta disyuntiva, planteada desde el punto de vista que concebimos a estos nuevos centros con una personalidad propia, que actuaría a modo de group mind. Antes de responder a nuestro interrogante, expondremos la delimitación semántica de cada una de las dos categorías: actores globales y actores locales.

Llamamos «actores globales» a aquellos actores sociales cuyas prácticas tienen un alcance al menos potencialmente y muchas veces efectivamente planetario -o al menos un alcance continental- lo cual visto en relación al alcance de las prácticas de los «actores locales» resulta al menos tendencialmente global, o casi-global. Estos actores globales son de muy diversos tipos y sus prácticas están orientadas por representaciones sociales e intereses disímiles y en ocasiones incluso contrapuestas, como por ejemplo organismos del sistema de la ONU; diversas agencias de los gobiernos de EEUU, Japón, Canadá y países de Europa occidental; fundaciones privadas y ONGs de distintos tipos, Objetivos de trabajo, y orientaciones (Amnesty Internacional, World Wildlife Foundation, Greenpeace, Fundación Rockefeller, Oxfam, Caritas, etc.); empresas de comunicación «culturales», y de entretenimiento; productores independientes de cine y video, periodistas, antropólogos, etc.

La denominación «actores locales» no debe interpretarse en el sentido de que estos actores sólo desarrollan sus prácticas a nivel local. En estos tiempos de globalización un número muy importante de actores locales desarrolla algún tipo de práctica trasnacional. El adjetivo «local sólo indica que sus principales intereses y actividades son geográficamente coincidentes con la localización de sus miembros, y que sus actividades trasnacionales son sólo complementarias.

Desde esta conceptualización, los CEPI son "actores locales", caracterizados por el desarrollo e intercambio de experiencias vividas de modernidad global (Tomlinson, 1999:113). Este intercambio en un futuro posibilitará allanar el camino para conceptualizar una cultura multicultural, que no esté basada en lo exótico del multiculturalismo o en la diversidad de las culturas acentuándose las desigualdades, sino en la inscripción y articulación de la hibridez de la cultura. De alguna manera, la "localidad cultural auténtica no existe ni existió nunca."

Este interrogante, aunque queda contestado dentro de los límites espaciales de todo artículo, supone para nosotros, como comunicólogos y como investigadores sociales, el auténtico atractivo de los CEPI, así como nos marca una de las líneas investigadoras en las que incidir en futuras aproximaciones a la comunicación institucional-intercultural.

\section{CONCLUSIÓN}

Los Centros de Participación e Integración del Inmigrante (CEPI) son una iniciativa pionera en Europa dadas las características conceptuales de las que parte. Se pueden considerar un espacio de negociación entre actores sociales en donde los fenómenos comunicativos son un elemento clave para la transformación social. La inicial situación de aislamiento del individuo y de los recursos que se ponen a su disposición (islas culturales) se transforman en ese proceso integrador en un estado de participación proclive a la hibridación cultural. La actividad comunicativa que se realiza en y por medio de estos centros cívicos puede entenderse, por sus peculiaridades, como un tipo particular de categoría socio-antropológica 
denominada "comunicación institucional-intercultural", donde aparece un agente activo (las administraciones públicas), un espacio en tránsito (CEPI) o una relación con la imagen de marca que la propia administración pretende transmitir, todo ello girando en torno al fenómeno comunicativo-intercultural que desde una actuación local abarca un fenómeno antropológico global.

\section{BIBLIOGRAFÍA}

BHABHA, H. K. (1994): The Location of Culture. London: Routledge.

CAPRIOTI, P. (1999): Planificación estratégica de la imagen corporativa. Barcelona: Ariel.

CARO, A. (2007): “Jean Baudrillard y la publicidad”, Pensar la Publicidad, vol. I, n. 2, pp. 131-146.

CLIFFORD, J. (1998): The Predicament of Culture; Twentieth-Century Ethnography, Literature and Art. Cambridge: Harvard University Press.

DOWLING, G. (1994): Corporate reputations: strategies for developing the corporate brand. Londres: Kogan Page.

GAONA, C. (2009): Ágora del impresionismo sociocultural. Madrid: Dykinson.

HANNERZ, U. (1998): Conexiones transnacionales: cultura, gente, lugares. Valencia: Cátedra.

HOFMANN, S. (1997): "Transculturation and Creolization: Concepts of Caribbean Cultural Theory" en Young, R.A (ed.): Latin American Postmodernisms. Amsterdam: Rodopi.

MARTÍNEZ, E. (2010): Los mensajes publicitarios analizados desde la comunicación y el derecho. Madrid: Universitas.

MARTÍNEZ, E., GAONA, C. y VIZCAÍNO-LAORGA, R. (2008): Comunicación institucional intercultural. Los CEPI, una iniciativa de la Comunidad de Madrid. Madrid: Observatorio de las Realidades Sociales y de la Comunicación.

PARADA, R. (1998): Derecho Administrativo I, parte general. Madrid-Barcelona: Marcial Pons.

LIE, R. (2003): "Espacios de comunicación intercultural", en La iniciativa de comunicación. 23 Conferencia AIECS, Barcelona, 21-26 junio 2002.

TOMLINSON, J. (1999): Globalization and Culture. Cambridge: Polity Press.

SENDÍN, J.C. y GAONA, C (2009): Por una ciencia comunicativa: Información, Cultura y Espacios Globales. Madrid: Universitas.

VIZCAÍNO-LAORGA, R, MARTÍNEZ, E. y GAONA, C. (2009): "Políticas de actuación desde la experiencia española. Convivencia y ciudad: la inmigración en España”, en Martínez, E. y Cerdá, P. (dir.) Bandas juveniles. Inmigración y Ciudad. Alicante: ECU. 


\section{Breve currículo:}

\section{Carmen Gaona Pisonero}

Diplomada Licenciada en Geografía e Historia, especialidad Antropología Social, y doctora en Sociología por la Universidad de Barcelona. Investigadora principal sobre las siguientes temáticas: Procesos de cambio y fenómeno migratorio; etnografía cultural, salud y desarrollo local, comunicación intercultural, Mass Media e inmigración. En la actualidad colabora en dos proyectos de investigación en el área de los mass media; uno sobre Medios de Comunicación e Inmigración financiado por la Comunidad de Madrid, junto a otro proyecto de ámbito europeo sobre Jóvenes e Internet.

\section{Esther Martínez Pastor}

Doctora en Ciencias de la Información por la Universidad Complutense de Madrid y Licenciada en Publicidad y RR. PP. por UCM y en Derecho por la UNED. Actualmente es profesora Contratada Doctora en la Facultad de Ciencias de la Comunicación de la Universidad Rey Juan Carlos de Madrid. Sus líneas de investigación se centran en la inmigración, la publicidad institucional de las Administraciones Públicas y la regulación de la publicidad. Ha sido co-investigadora principal del proyecto, RECUERDO financiado por la Consejería de Inmigración de la Comunidad de Madrid; la "Proyección de la diversidad cultural y de la integración de la inmigración en la Región de Murcia a través de la publicidad" financiado por la Consejería de Trabajo y Política Social de la Comunidad Autónoma de Murcia; "Imagen, comunicación e inmigración” financiado por la Fundación Séneca.

\section{Ricardo Vizcaíno-Laorga}

Profesor Contratado Doctor, sus líneas de investigación abarcan los usos y riesgos de Internet por parte de menores, la Discapacidad y la inmigración en relación con comunicación. Ha sido coinvestigador principal de proyectos como ULISES (desarrollo de Avatares para discapacitados auditivos) o CALISTO (recursos tecnológicos para mayores mediante la TDT). Igualmente ha sido integrante de otros como HERMES (cumplimiento de normativas de subtitulado en TDT), SUBSORDIG (subtitulado para sordos). Dentro de la línea de inmigración ha participado en la organización de las Jornadas COCTEL (publicidad e inmigración) y en proyectos como RECUERDO.

*(Los currículos no están incluidos en el cómputo de páginas del artículo) 